\title{
Scheduling Strategies for Coordinated Multi-Point Systems
}

\author{
T. F. Maciel, E. M. G. Stancanelli, J. C. M. Feitosa, W. C. Freitas Jr., and F. R. P. Cavalcanti
}

\begin{abstract}
Alike Multiple Input Multiple Output systems, Coordinated Multi-Point (CoMP) has recently raised in the $3^{\text {rd }}$ Generation Partnership Project Long Term Evolution context as a promising solution to improve Signal-to-Noise Ratio levels and, consequently, the system performance compared to conventional cellular networks. CoMP systems decrease the average access distances between Antenna Port (AP) and User Equipment (UE) and, at the same time, they allow for handling coverage, decreasing transmit powers, and/or increasing system capacity. Together with the inherent resource granularity of Orthogonal Frequency Division Multiple Access, advanced Radio Resource Management algorithms can be developed for these systems. In this work, we focus on the Resource Allocation subproblem of determining the most suitable set of APs to serve the UEs selected according to certain allocation criteria. Our analyses have shown that increasing the number of APs serving a given UE brings only small performance gains, but may cause a significant increase of complexity and signaling.
\end{abstract}

Index Terms-Coordinated Multi-Point, Antenna Port Selection, Resource Allocation Algorithm.

\section{INTRODUCTION}

$\mathbf{R}$ ECENTLY, advanced antenna architectures attracted a lot of interest as a means to improve the performance of conventional cellular networks. In particular, Coordinated Multi-Point (CoMP) appears in the $3^{\text {rd }}$ Generation Partnership Project (3GPP) Long Term Evolution (LTE) context as a promising architecture. CoMP systems are composed of several geographically distributed Antenna Ports (APs) connected through a fast backhaul to an Enhanced Node B (eNB), which might also have an AP [1]-[3], as illustrated in Fig. 1.

The actual number and placement of APs depend on the geographical densities of User Equipments (UEs) and services, planned coverage, Quality of Service (QoS) requirements, propagation environment, among other aspects that must be taken into account during the network planning stage. CoMP systems decrease the average access distances between APs and UEs and, at the same time, they allow for handling the coverage efficiently, decreasing transmit powers, and/or increasing system capacity.

A CoMP system can also be viewed as a distributed Multiple Input Multiple Output (MIMO) system, thus offering similar advantages over the Single Input Single Output (SISO) systems, such as improved capacity and link reliability due to spatial multiplexing and spatial diversity, respectively. The fast backhaul makes easier to the eNB to work as a central

This work was supported by the Research and Development Center, Ericsson Telecomunicações S.A., Brazil, under EDB/UFC.22 Technical Cooperation Contract.

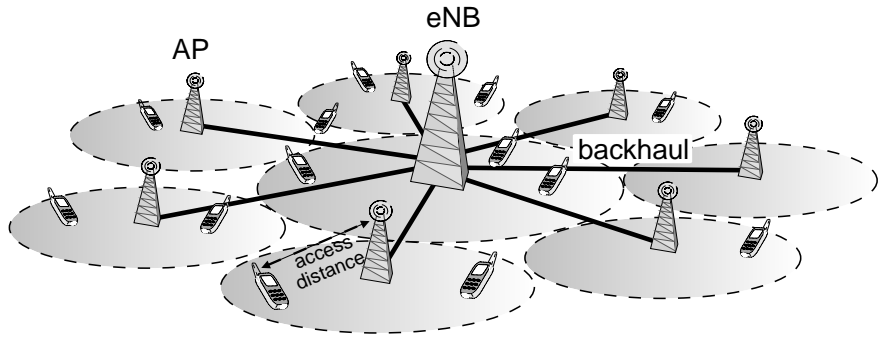

Fig. 1. Basic composition of a CoMP system.

controller and to employ cooperative transmission/reception techniques.

By exploiting the geographical distribution of APs and UEs, the possibilities for managing radio resources are increased in CoMP systems. In particular, by combining the inherent resource granularity of Orthogonal Frequency Division Multiple Access (OFDMA) with the flexible CoMP architecture, advanced Radio Resource Management (RRM) algorithms can be developed to control the power allocation, the assignment of subcarriers to APs and UEs, the selection of APs for transmission, and UEs prioritization. For CoMP systems, constraints on QoS and radio resource usage might complicate finding optimal Resource Allocation (RA) solutions, while constraints on signaling between APs and the eNB might be alleviated due to the fast backhaul assumption.

In this work, we focus on the RA subproblem of determining the most suitable set of APs to serve the UEs selected according to certain allocation criteria. The rest of this work is organized as follows. Section II presents the adopted CoMP model. In section III, we introduce the RA problem investigated in this work followed by the description of the employed allocation criteria. Section IV discusses some CoMP performance results. Finally, in section V, some conclusions are drawn.

\section{COMP SySTEM MODEL}

We consider the downlink of a single CoMP cell with a number $M$ of APs, indicated by $m=1,2, \ldots, M$, which are controlled by an eNB.

The APs are placed over the coverage area at positions determined using Particle Swarm Optimization (PSO) [4]. PSO is a heuristic-based on artificial life and evolutionary computation. It is basically consisting on a population of particles that adapt by stochastically returning towards previously successful regions in the search space. PSO is iterative and each particle has a position - representing a possible solution of the problem -, a velocity, and a best 
position. Moreover, there is a global best position, which is the best among all best positions. The efficiency of each particle is called fitness and is used to determine local and global best positions. PSO updates these parameters iteratively as to move towards the global optimum, i.e., the overall best position.

In this work, the fitness function has been defined as the lowest Signal-to-Noise Ratio (SNR) among the a large number of UEs uniformly distributed over the cell area so that the placement of the APs is performed as maximize this function.

The advantages of PSO over some other evolutionary optimization techniques are not only its implementation easiness, but also the reduced number of parameters to adjust.

The CoMP system serves a number $J$ of single-antenna UEs, indicated by $j=1,2, \ldots, J$, which are uniformly distributed over the coverage area.

The average path loss $G_{j, m}^{\mathrm{pl}}(d)$ in dB for a UE $j$ distant of $d_{j, m}$ kilometers of an AP $m$ is modeled according to the Okumura-Hata's model of [5] as

$$
G_{j, m}^{\mathrm{pl}}(d)=128.1+37.6 \log _{10}\left(d_{j, m}\right),
$$

and denoted in linear scale by $g_{j, m}^{\mathrm{pl}}$, where the dependency on $d_{j, m}$ has been omitted for simplicity of notation.

The shadowing $G_{j, m}^{\mathrm{sh}}$ in dB affecting the link between the AP $m$ and UE $j$ is modeled as a lognormal random variable with standard deviation $\sigma_{S}$ [5]. In linear scale, the shadowing is denoted $g_{j, m}^{\mathrm{sh}}$. Frequency selectivity is modeled using a tapped-delay channel model and short-term fading in each tap is modeled using Jakes' model [6].

The considered CoMP system employs OFDMA and considers a number $N$ of subcarriers, indicated by $n=$ $1,2, \ldots, N$. Each subcarrier can be allocated individually to a link between an AP $m$ and a UE $j$. As the channel coherence bandwidth is assumed to be much larger than the subcarrier bandwidth, we have flat fading on each subcarrier. The absolute value of the complex channel coefficient $h_{j, m, n}$ for the link between the AP $m$ and the UE $j$ on subcarrier $n$ is denoted in linear scale as $g_{j, m, n}^{\mathrm{ff}}$.

The eNB has a total transmit power $p$ that is divided among the system subcarriers by means of a resource allocation algorithm, and thereafter $p_{n}$ denotes the power allocated to subcarrier $n$. On each subcarrier $n$, the fraction $p_{m, n}$ of the power $p_{n}$ that is allocated to the AP $m$ is decided by the eNB, which is assumed to ideally have perfect knowledge about the channels of all UEs.

Denoting by $\sigma^{2}$ the average Additive White Gaussian Noise (AWGN) power, the SNR $\gamma_{j, m, n}$ of the link between the AP $m$ and the UE $j$ at subcarrier $n$ is given by

$$
\gamma_{j, m, n}=\frac{p_{m, n} g_{j, m}^{\mathrm{pl}} g_{j, m}^{\mathrm{sh}} g_{j, m, n}^{\mathrm{ff}}}{\sigma^{2}}=\frac{p_{m, n} g_{j, m, n}}{\sigma^{2}},
$$

where $g_{j, m, n}$ is the gain of the link between AP $m$ and UE $j$ on subcarrier $n$.

\section{Resource Allocation}

For the considered CoMP system, let $\mathcal{J}=\{1, \ldots, J\}$, $\mathcal{M}=\{1, \ldots, M\}$, and $\mathcal{N}=\{1, \ldots, N\}$ denote the sets of UEs, APs, and subcarriers, respectively. Also, let $x_{j, m, n}$ be a binary variable indicating whether AP $m$ sends data to the UE $j$ through subcarrier $n$ with power $p_{j, m, n}$. The variables $x_{j, m, n}$ and $p_{j, m, n}, \forall j, m, n$, are organized in an allocation set $\mathcal{X}$ and a power set $\mathcal{P}$, respectively.

\section{A. AP Selection Problem}

In this work, we restrict our investigations to the problem of selecting $M^{\prime}$ among the $M$ existing APs for coordinated transmission on each individual subcarrier. Subcarrier reuse is not allowed and no restrictions are imposed initially onto the bandwidth shares allocated to each AP and to each UE. Let $\gamma_{j, n}$ denote the SNR perceived by UE $j$ resulting from the coordinated transmission of the $M^{\prime}$ APs selected for this UE and let $r_{j, n}\left(\gamma_{j, n}\right)$ denote the rate of UE $j$ on subcarrier $n$. Considering the previous definitions and considering the objective of maximizing the sum rate of the system, the problem of selecting the best $M^{\prime}$ APs for coordinated transmission can be formulated as

$$
\left\{\mathcal{P}^{\star}, \mathcal{X}^{\star}\right\}=\underset{\{\mathcal{P}, \mathcal{X}\}}{\arg \max }\left\{\sum_{\substack{j \in \mathcal{J}, n \in \mathcal{N}}} r_{j, n}\right\},
$$

subject to

$$
\begin{aligned}
& \sum_{m \in \mathcal{M}} \sum_{j \in \mathcal{J}} x_{j, m, n} \leq 1, \forall n, \\
& \sum_{m \in \mathcal{M}} x_{j, m, n} \leq M^{\prime}, \forall j, n, \\
& \sum_{\substack{j \in \mathcal{J} \\
m \in \mathcal{M}, n \in \mathcal{N}}} p_{j, m, n} \leq p, \\
& p_{j, m, n} \geq 0, \forall j, m, n,
\end{aligned}
$$

Considering perfect channel knowledge, problem (3) can be seen as an antenna selection problem [7], [8]. Because perfect channel knowledge is assumed at the eNB, it can perform Maximum Ratio Transmission (MRT) which corresponds to apply a spatial transmit matched filter using the APs as an antenna array [9].

Let $(\cdot)^{\mathrm{T}}$ and $(\cdot)^{\mathrm{H}}$ denote transposition and conjugate-transposition, respectively, and let $\mathcal{M}^{\prime}=$ $\left\{1,2, \ldots, M^{\prime}\right\}$ denote the set of APs selected for transmission. Also consider a given UE $j$ and subcarrier $n$, whose indices will be omitted in the sequel for simplicity of notation. Then, let $\mathbf{h}=\left[\begin{array}{llll}h_{1} & h_{2} & \ldots & h_{M^{\prime}}\end{array}\right]$ denote the complex vector channel of the $M^{\prime}$ selected APs to the UE $j$ on subcarrier $n$. Considering MRT, a symbol $s$ is weighted at the APs with a weighting vector $\mathbf{w}=\left[\begin{array}{llll}w_{1} & w_{2} & \ldots & w_{M^{\prime}}\end{array}\right]^{\mathrm{T}}$, sent through the vector channel $\mathbf{h}$, corrupted by the AWGN noise sample $\nu$, and received by the UE $j$, which estimates the symbol $s$ as

$$
\hat{s}=\mathbf{h w}^{\mathrm{H}} s+\nu .
$$

Denoting by $\|\cdot\|_{2}$ the Euclidean norm of a vector and considering MRT, we have that

$$
\mathbf{w}=\frac{\mathbf{h}^{\mathrm{H}} \sqrt{p_{n}}}{\|\mathbf{h}\|_{2}} .
$$


Then, considering an ideally matched receive filter, the SNR $\gamma$ perceived by UE $j$ on subcarrier $n$ is given by

$$
\gamma=\frac{p_{n}\|\mathbf{h}\|_{2}^{2}}{\sigma^{2}}
$$

\section{B. UE Prioritization}

Considering MRT, the rate of any individual UE on a given subcarrier is maximized by selecting the $M^{\prime}$ APs with highest channel gains $g_{j, m, n}$ [7], [8]. Due to constraint (3b), only a single UE is scheduled per subcarrier. Thus, problem (3) describes the maximization of the sum rate of a single-user OFDMA Multiple Input Single Output (MISO) system. As a direct extension of the same problem in single-user OFDMA SISO systems, it is well-known that the sum rate of the considered MISO system is maximized by assigning each subcarrier $n$ to the UE $j^{\star}$ with the highest channel norm, i.e.,

$$
j^{\star}=\underset{j \in \mathcal{J}}{\arg \max }\left\{\|\mathbf{h}\|_{2}^{2}\right\},
$$

and by allocating afterwards power to the subcarriers according to the Waterfilling (WF) algorithm [10]. Although optimal, WF is not considered in this work, but Equal Power Allocation (EPA) among the subcarriers. EPA is simpler and performs only marginally worse than WF, especially for high SNRs [11].

Indeed, (7) describes an allocation criterion, which will be referred in this work as the Rate Maximization (RM) criterion. In spite of leading to a high sum rate to the system, the RM criterion results in quite unfair rate distributions among the UEs, which might be undesired in specific scenarios.

In order to provide some degree of fairness for the rate distribution among the UEs, two additional criteria are considered in this work, namely Fair Resource (FR) and Proportional Fair (PF), which are described in the sequel.

For the FR criterion, subcarriers are assigned sequentially to the UEs in rounds, where a round finishes when all UEs got a subcarrier assigned. Within a round, the assignment of the current subcarrier to a UE follows the same criterion described by (7). However, only UEs that did not get a subcarrier in the current round are eligible for subcarrier assignment. We denote by $\mathcal{J}^{\prime}$ the set of UE eligible for subcarrier assignment and by $\bmod (a, b)$ the remainder of the division of $a$ by $b$.

For the PF criterion, subcarriers are assigned sequentially to the UEs with the best ratio between instantaneous rate $r_{j, m}$ and average rate $\bar{r}_{j}$. The average rate $\bar{r}_{j}$ is updated every time all subcarriers are allocated. The RA algorithm considering the RM, FR and PF criterion are shown in Table I.

\section{Analyses AND Results}

The RA subproblem described in section III-A is analyzed herein through simulations. For this purpose, a system-level simulation tool has been implemented based on the CoMP and propagation models described in section II.

We consider a CoMP cell with a coverage radius $R$ of $500 \mathrm{~m}$. For the shadowing, a standard deviation $\sigma_{S}=8 \mathrm{~dB}$ is considered. The fading assumes an average speed of $3 \mathrm{~km} / \mathrm{h}$ in a typical urban power-delay profile: TU3 [12]. The CoMP
TABLE I

RA ALGORITHMS.

\begin{tabular}{l}
\hline \multicolumn{1}{c}{ RM criterion } \\
\hline 1) Set $x_{j, n, m}=0, \forall j, n . m$ \\
2) For $n=1$ to $N$ \\
a) Set $j^{\star}=\underset{j \in \mathcal{J}}{\arg \max }\left\{\|\mathbf{h}\|_{2}^{2}\right\}$ \\
b) Set $x_{j^{\star}, n, m^{\prime}}=1, \forall m^{\prime} \in \mathcal{M}^{\prime}$, and set $x_{j^{\star}, n, m}=0, \forall m \in \mathcal{M} \backslash \mathcal{M}^{\prime}$ \\
\hline \multicolumn{1}{c}{ FR criterion } \\
\hline 1) Set $x_{j, n, m}=0, \forall j, n . m$ \\
2) Set $n=1$ and set $\mathcal{J}^{\prime}=\mathcal{J}$ \\
3) do \\
a) Set $j^{\star}=\underset{j \in \mathcal{J}^{\prime}}{\arg \max }\left\{\|\mathbf{h}\|_{2}^{2}\right\}$ \\
b) Set $x_{j^{\star}, n, m^{\prime}}=1, \forall m^{\prime} \in \mathcal{M}^{\prime}$, and set $x_{j^{\star}, n, m}=0, \forall m \in \mathcal{M} \backslash \mathcal{M}^{\prime}$ \\
c) Set $\mathcal{J}^{\prime}=0 j^{\star} \backslash\left\{j^{\star}\right\}$ \\
d) If $\mathcal{J}^{\prime}=\emptyset, \operatorname{set} \mathcal{J}^{\prime}=\mathcal{J}$ \\
e) Set $n=\bmod (n, N)+1$ \\
4) loop
\end{tabular}

1) Set $x_{j, n, m}=0, \forall j, n . m$

2) For $n=1$ to $N$
a) Set $j^{\star}=\underset{j \in \mathcal{J}}{\arg \max }\left\{\frac{r_{j, n}}{\bar{r}_{j}}\right\}$
b) Set $x_{j^{\star}, n, m^{\prime}}=1, \forall m^{\prime} \in \mathcal{M}^{\prime}$, and set $x_{j^{\star}, n, m}=0, \forall m \in \mathcal{M} \backslash \mathcal{M}^{\prime}$

considers a carrier frequency $f_{c}$ of $2 \mathrm{GHz}$ and a number $N=100$ subcarriers spaced of $\Delta f=15 \mathrm{kHz}$. EPA among subcarriers is considered. Moreover, we assume perfect knowledge about the channels of all UEs at the eNB.

The eNB coordinates the operation of a variable number $M$ of APs, whose topologies have been established using PSO. Considering the fitness function mentioned in section II, the PSO has lead to circular topologies with APs placed at the same radius around the cell center and at angles equally spaced. The radius of the APs are listed in Table II. A SISO system is characterized when the number $M$ of APs is just 1, which is then positioned at the cell center.

TABLE II

COMP TOPOLOGY: RADIUS (IN M) OF AP DISTRIBUTION AROUND THE CENTER OF THE COVERAGE AREA.

\begin{tabular}{l|c|c|c}
\hline Number $\boldsymbol{M}$ of APs & 4 & 5 & 7 \\
\hline Radius $\boldsymbol{R}$ in $\mathbf{~ m}$ & 350 & 370 & 335 \\
\hline
\end{tabular}

Simulations are organized in snapshots, during which path loss and shadowing are assumed to remain constant for all UEs, but the time variations of short-term fading are considered. In order to capture the fading dynamics, each snapshot takes $1 \mathrm{~s}$, which is longer than 10 times the channel coherence time. In order to capture the impact of long term propagation effects on the system performance, a large number of snapshots is considered.

A variable number $J$ of UEs are uniformly distributed over the coverage radius of the CoMP cell. It is assumed that UEs make use of a best-effort service and always have data to receive. Average rates of UEs are assumed to be updated at each Transmission Time Interval (TTI), i.e., each $1 \mathrm{~ms}$. In Table III, the most important parameters considered in the simulations are summarized.

The available modulations are Binary Phase-Shift Keying 
TABLE III

SIMULATION PARAMETERS.

\begin{tabular}{l|c|c}
\hline \hline Parameter & Symbol & Value \\
\hline \hline Coverage radius & $R$ & $500 \mathrm{~m}$ \\
\hline Carrier frequency & $f_{c}$ & $2 \mathrm{GHz}$ \\
\hline Number of subcarriers & $N$ & 100 \\
\hline Subcarrier spacing & $\Delta f$ & $15 \mathrm{kHz}$ \\
\hline Shadowing standard deviation & $\sigma_{S}$ & $8 \mathrm{~dB}$ \\
\hline Channel profile & - & $\mathrm{TU} 3[12]$ \\
\hline Snapshot duration & - & $1 \mathrm{~s}$ \\
\hline Transmission Time Interval & - & $1 \mathrm{~ms}$ \\
\hline Number of APs & $M$ & 1,4 or 7 \\
\hline Number of selected APs & $M^{\prime}$ & 1 to $M$ \\
\hline Number of UEs & $J$ & $2,4,8$ or 16 \\
\hline Scheduling algorithm & - & $\mathrm{RM}$, FR, PF \\
\hline
\end{tabular}

(BPSK) and 4-, 16- and 64-Quadrature Amplitude Modulation (QAM), and the link adaptation is performed based on SNR thresholds that ensure a target Symbol Error Rate (SER) of $10^{-6}$. The values of SNR threshold used are summarized in Table IV [13]. UEs do not receive data when their perceived SNR is below $10 \mathrm{~dB}$.

TABLE IV

SNR THRESHOLDS FOR LINK ADAPTATION.

\begin{tabular}{l|c|c|c|c}
\hline Modulation & BPSK & 4-QPSK & 16-QAM & 64-QAM \\
\hline SNR threshold in dB & 10.0 & 13.5 & 21.5 & 27.8 \\
\hline
\end{tabular}

Initially, it is important to investigate the improvement in the SNR levels obtained by a CoMP system compared to a SISO system. Furthermore, the notation $M^{\prime} / M$ will be used to characterize CoMP scenarios in terms of the numbers of selected and available APs, respectively. In Fig. 2, the Cumulative Distribution Function (CDF) of the SNR values perceived by all UEs and by the scheduled UEs in an 1/1 (i.e., SISO) and in a 4/4 CoMP system are shown. Both systems have the same total transmit power $p$. The RM criterion is used and a total of 16 UEs is considered.

We can observe in Fig. 2 that, at the $10^{\text {th }}$ percentile, an SNR gain between 5 and $9 \mathrm{~dB}$ is obtained by the $4 / 4$ CoMP system compared to the SISO one. This gain results from the improved coverage in the CoMP system due to the shorter access distances. Regarding the scheduled UE, it can be noted that an even larger gain is obtained compared to the SNR values of all UEs in the system. This gain results from

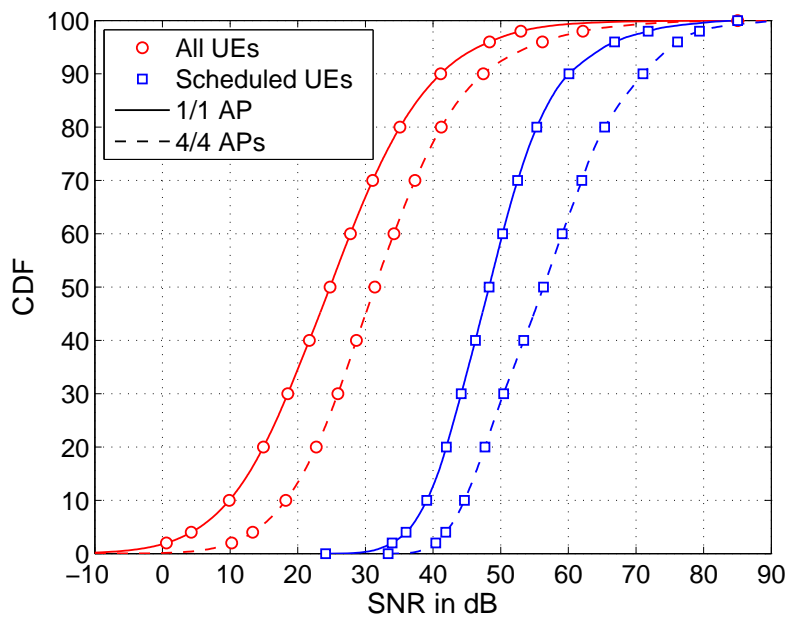

Fig. 2. CDF of SNR values for a SISO and $4 / 4$ CoMP system. $J=16$ UEs. RM criterion.

the multi-user diversity exploited when considering the RM criterion, which leads to the selection on each subcarrier of the UE with the best channel condition.

Alternatively, this SNR gain obtained through CoMP can be used to save transmit power while the same coverage radius is kept. For this purpose, the link budget that will be employed from now on has been designed to set the total transmit power as to assure the minimum SNR required by the BPSK modulation with a $90 \%$ confidence level regarding the shadowing. Depending on the APs placement topology, that minimum SNR may occur either at the center or edge of coverage area. Regarding a configuration with lower power and interference levels in a macroscopic context, from now on we also consider a penalty of $10 \log _{10} M \mathrm{~dB}$ on the link budget, which roughly corresponds to the gain of the distributed antenna array.

Fig. 3 shows the CDF of the system throughput for the RM, FR, and PF criteria for different loads in number of UEs and considering an 1/4 CoMP topology. As we can note, RM provides the best system throughput once it is the most favored by the multi-user diversity. That is, the higher the number of UEs, the better the system throughput. The multi-user diversity is especially important in CoMP systems because

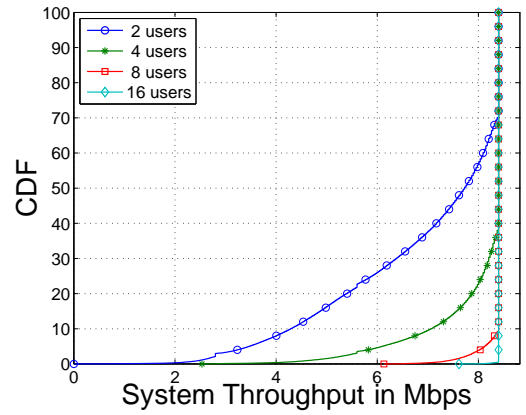

(a) Rate Maximization

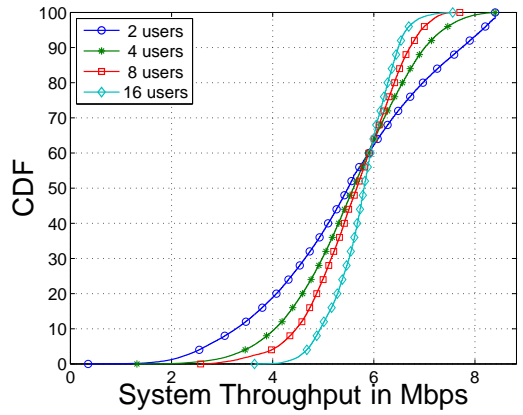

(b) Fair Resource.

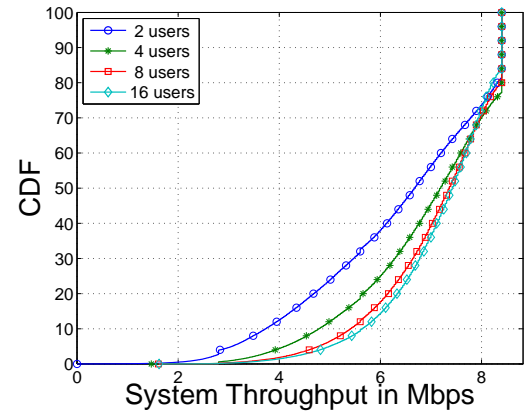

(c) Proportional Fair.

Fig. 3. System throughput at fixed loads for algorithms 1/4 CoMP system. 
the geographical distribution of APs increases the chance of existing UEs with excellent link to one or more APs. The multi-user diversity benefits also FR and PF schedulers, but for them another influencing factor takes place: the resource sharing. Resource sharing means that the UEs in worst channel conditions also compete for radio resources, and, for the sake of fairness, they are often selected even for a relatively inefficient transmission. The throughput losses due to resource sharing are clearer to FR than to PF, since the latter considers a trade-off between the instantaneous UEs' rates, which favors UEs in good channel conditions, and the average UEs' rates, which introduces fairness.

Another relevant effect to be investigated is the combining gain obtainted by increasing the number $M^{\prime}$ of APs selected for coordinated transmission. In Fig. 4, CDFs of the system throughput are presented for a topology of $M=7$ APs and considering the RM criterion. It is worthy noting that the system throughput gain due to combining diversity is not very significant. However, one incurs increased complexity and signaling when increasing $M^{\prime}$ because more channel state information about more links must be transferred through the air interface and through the backhaul.

Fig. 5 extends this analysis to compare different loads and allocation criteria. The $10^{\text {th }}$ percentile of system throughput for a topology with $M=7$ APs is presented in Fig. 5(a) for a varying the number $M^{\prime}$ of the selected APs. Similarly to the previous results, the RM criterion leads to higher throughputs than the PF one, which outperforms the FR criterion in terms of throughput. Moreover, due to the multi-user diversity, the performance is improved with increasing number $J$ of UEs.

In terms of fairness, opposite results might be reached. For this purpose, we evaluate the distribution of the average UE throughput, which gives some insight on the disparities in allocating radio resources among the APs. In Fig. 5(b), the $10^{\text {th }}$ percentile of average UE throughput for the 7-APs topology is presented varying the number of the selected APs. Now, using the RM criterion performs the worst, being not able to provide even a $200 \mathrm{kbps}$ rate for a low load (2 UEs). The FR obtains at the $10^{\text {th }}$ percentile an average user throughput between 1.0 and 1.3 Mbps for load of 4 UEs, and between 2.0 and 2.5 Mbps for 2 UEs. Due to its characteristic of taking advantage of good channel conditions, the PF was the best, reaching to throughputs close to 2.8 and $1.5 \mathrm{Mbps}$ for load of 2 and 4 UEs, respectively.

\section{CONCLUSiOnS}

In this work, we evaluated by means of simulations the performance of a Coordinated Multi-Point (CoMP) system, which considered different topologies determined offline using the Particle Swarm Optimization technique and considering the maximization of the lowest Signal-to-Noise Ratio (SNR) as fitness function to optimize the positions of the Antenna Ports (APs). Considering these pre-established AP topologies, we investigated the Resource Allocation (RA) subproblem of determining the most suitable set of APs for coordinated transmission to the User Equipments (UEs). Rate Maximization (RM), Fair Resource (FR) and Proportional Fair

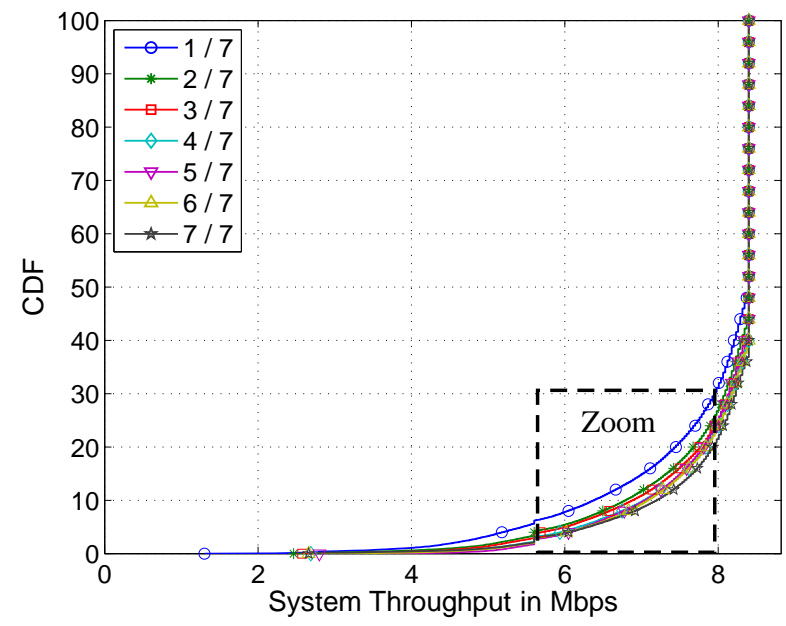

(a) Normal view.

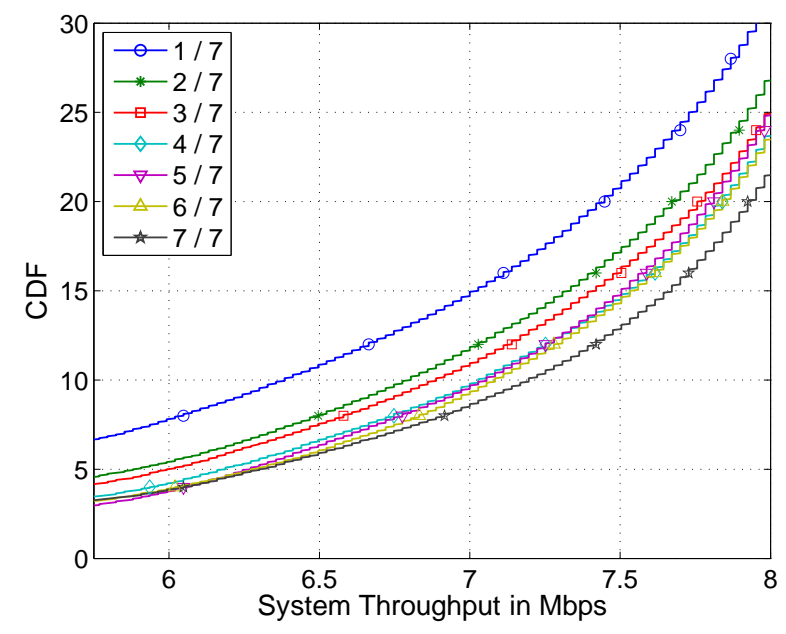

(b) Zoomed view.

Fig. 4. System throughput in a 7-APs topology for different number of APs selectable. RM criterion. $J=2$ UEs.

(PF) allocation criteria have been employed for allocating resources to UEs and the peculiarities of these criteria have been discussed.

Regarding the allocation criteria, only the RM criterion invariably provided multi-user diversity gains significantly improving the system throughput. FR and PF can also take advantage of multi-user diversity, but in smaller scale. Moreover, they aim at providing some degree of throughput fairness to the UEs by performing resource sharing even with UEs perceiving very poor channel conditions. Consequently, the FR and PF criteria lead to lower total system throughput values than the RM criterion. On the other hand, the RM criterion is the only unfair one, presenting high dispersion of average UE throughput.

Due to the lower average access distances, better coverage could be reached in the CoMP system compared to the Single Input Single Output (SISO) one, with both systems having the same total transmit power. We observed that increasing the number of APs yielded better performance even when penalties on the total transmit power were applied as more 


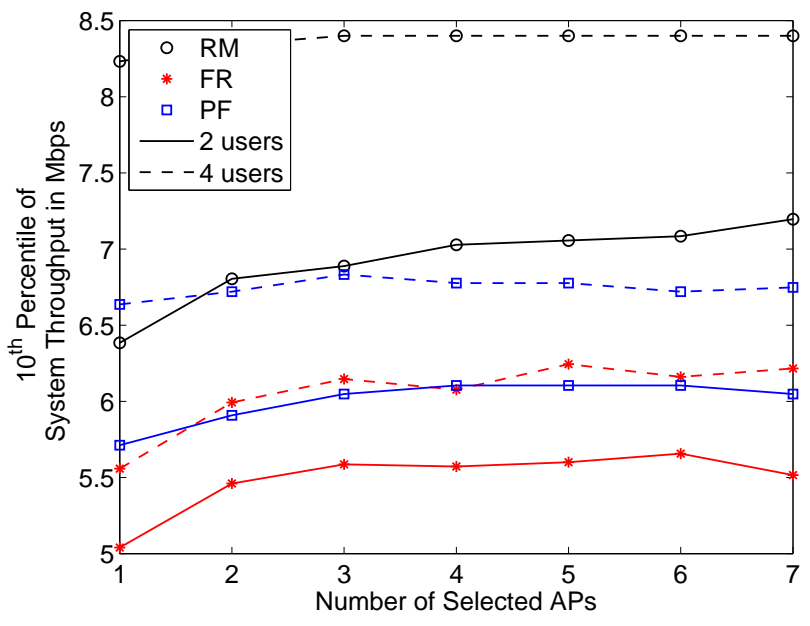

(a) System throughput.

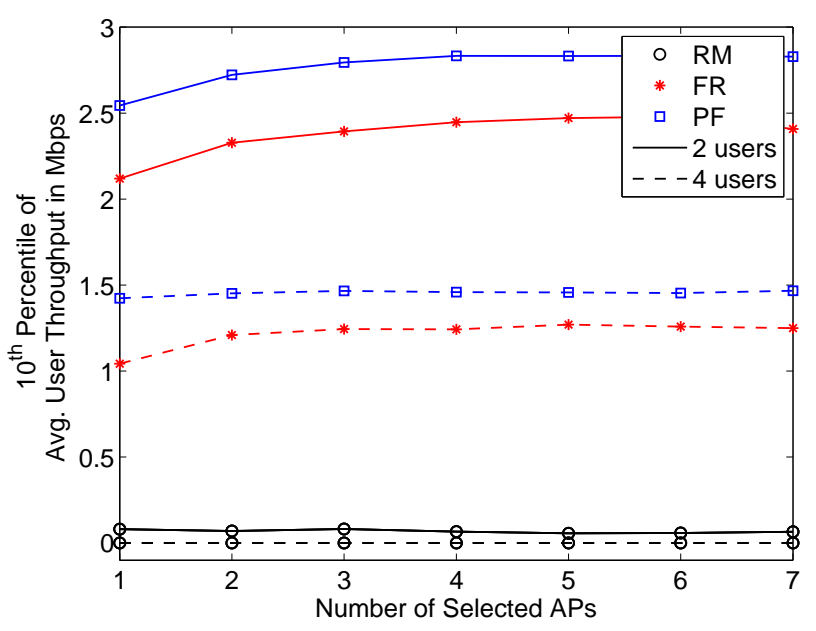

(b) Average UE throughput.

Fig. 5. $10^{\text {th }}$ percentile of system and average UE throughputs in a 7-APs topology for different number of selectable APs.

APs were employed. On the other hand, increasing the number of APs seemed not so advantageous, since the perceived combining gains were not very significant. Using a few APs can already provide similar results compared to the usage of all APs and might represent saves in terms of complexity and signaling.

\section{REFERENCES}

[1] A. A. M. Saleh, A. J. Rustako Jr., and R. S. Roman, "Distributed antennas for indoor radio communications," IEEE Transactions on Communications, vol. 35, pp. 1245-1251, 1987.

[2] M. V. Clark, T. M. Willis, L. J. Greenstein, A. J. Rustako Jr., V. Ercegt, and R. S. Roman, "Distributed versus centralized antenna arrays in broadband wireless networks," in Proceedings of the IEEE Vehicular Technology Conference (VTC), 2001.

[3] H. Hu, Y. Zhang, and J. Luo, Eds., Distributed antenna systems: open architecture for future wireless communications, 1st ed., ser. Wireless networks and mobile communications. Auerbach, Jul 2007.

[4] J. Kennedy and R. Eberhart, "Particle swarm optimization," in Proceedings of the 1995 IEEE International Conference on Neural Networks, Vol. 4, 1995, pp. 1942-1948.
[5] UMTS, "Selection procedures for the choice of radio transmission technologies of the UMTS," UMTS, UMTS 101.112 v.3.2.0, Tech. Rep., Apr. 1998

[6] W. C. Jakes, Microwave mobile communications, 1st ed. New York: John Wiley \& Sons, 1974.

[7] S. Sanayei and A. Nosratinia, "Antenna selection in MIMO systems," IEEE Communications Magazine, vol. 42, no. 10, pp. 68-73, Oct. 2004.

[8] - "Capacity of MIMO channels with antenna selection," IEEE Transactions on Information Theory, vol. 53, no. 11, pp. 4356-4362, Nov. 2007.

[9] M. Joham, W. Utschick, and J. A. Nossek, "Linear transmit processing in MIMO communications systems," IEEE Transactions on Signal Processing, vol. 53, no. 8, pp. 2700-2712, Aug. 2005.

[10] D. P. Palomar and J. R. Fonollosa, "Practical algorithms for a family of waterfilling solutions," IEEE Transactions on Signal Processing, vol. 53, no. 2, pp. 687-695, Feb. 2005.

[11] D. Tse and P. Viswanath, Fundamentals of wireless communications, 1st ed. Cambridge University Press, 2005.

[12] 3GPP, "Deployment aspects," $3^{\text {rd }}$ Generation Partnership Project, Tech. Rep. TS 25.943 V8.0.0 - Release 8, Dec 2008. [Online]. Available: http://www.3gpp.org

[13] J. G. Proakis, Digital Communications, 4th ed., ser. Electrical Engineering Series. McGraw-Hill, 2001. 